\title{
Programas de vigilancia de recién nacidos de riesgo: experiencias municipales brasileñas
}

\author{
Newborn risk surveillance programs: Brazilian \\ municipal experiences
}

\section{Programas de vigilância de recém nascidos de risco: experiências municipais brasileiras}

\author{
Deisi Cardoso Soares ${ }^{1}$, Luiz Augusto Facchini², Sidnéia Tessmer \\ Casarin $^{3}$, Elaine Thumé ${ }^{4}$
}

\begin{abstract}
${ }^{1}$ Enfermeira. Doutora em Ciências. Professora Adjunta do Departamento de Enfermagem da Universidade Federal de Pelotas. Correo electrónico: soaresdeisi@gmail.com

${ }^{2}$ Médico. Doutor em Ciências Médicas. Professor Associado do Departamento de Medicina Preventiva e Social e dos Programas de Pós-Graduação em Epidemiologia e em Enfermagem da Universidade Federal de Pelotas. Correo electrónico: luizfacchini@gmail.com
\end{abstract}

${ }^{3}$ Enfermeira. Mestre em Enfermagem. Professora Assistente do Departamento de Enfermagem da Universidade Federal de Pelotas. Doutoranda do Programa de Pós-Graduação em Enfermagem da Universidade Federal de Pelotas. Correo electrónico: stcasarin@gmail.com

${ }^{4}$ Enfermeira. Doutora em Epidemiologia. Professora Adjunta do Departamento de Enfermagem e do Programa de Pós-Graduação em Enfermagem da Universidade Federal de Pelotas. Correo electrónico: elainethume@gmail.com

Cómo citar este artículo en edición digital: Soares. D.C., Facchini, L.A., Casarin, S.T. E Thumé, E.

(2019). Programas de vigilancia de recién nacidos de riesgo: experiencias municipales brasileñas.

Cultura de los Cuidados (Edición digital), 23(53). Recuperado de http://dx.doi.org/10.14198/cuid.2019.53.30

Correspondencia: Deisi Cardoso Soares.Rua Gomes Carneiro, n. 1. Centro. Faculdade de Enfermagem. CEP: 96010-610. Pelotas-RS

Correo electrónico de contacto: rdediego2@us.es

Recibido: 10/10/2018; Aceptado: 11/01/2019.es



\begin{abstract}
Objective: To identify newborns at risk surveillance programs for infant mortality within the Brazil, highlighting the operalisation strategies and inclusion criteria.
\end{abstract}

Methods: A literature review in the database dados Medical Literature Analysis and Retrieval System Online, Latin American and Caribbean Literature in Health Sciences and the portal of electronic journals Scientific Electronic Library Online. The articles that were included addressed the description of the criteria for inclusion in programs for the surveillance of newborns at risk and their operationalization. Exclusion criteria were editorials or letters to the editor, opinion articles, summaries presented in annals, theses or dissertations.

Results: Seventeen articles described the eight programs experience, in four brazilian states, in the Southeast and South. The identification of newborns at risk was done through interviews in hospitals or Live Birth 
Declaration. The criteria used included biological and social risks, especially prematurity, low birth weight, maternal age and education.

Conclusion: Articles about the theme are scarce and among the risk factors identified, most are likely to change in actions related to prenatal care and health education.

Keywords: Newborn, risk factors, public health surveillance.

\section{RESUMO}

Objetivo: Identificar os programas brasileiros de vigilância de recém-nascidos de risco para mortalidade infantil, destacando as estratégias de operacionalização e os critérios para inclusão.

Método: Revisão integrativa da literatura na base de dados Medical Literature Analysis and Retrieval System Online, Literatura Latino-americana e do Caribe em Ciências da Saúde e no portal de revistas eletrônicas Scientific Eletronic Library Online. Foram incluídos os artigos que abordavam a descrição dos critérios de inclusão em programas de vigilância de recém-nascido de risco e a operacionalização dos mesmos. Não foram incluídos os editoriais ou cartas ao editor, artigos de opinião, resumos apresentados em anais, teses ou dissertações.

Resultados: Dezessete artigos descreveram a experiência de oito programas, em quatro estados brasileiros, das regiões Sul e Sudeste. A identificação dos recém-nascidos era através de entrevistas nas maternidades ou da Declaração de Nascidos Vivos. Os critérios utilizados incluáam riscos biológicos e sociais, com destaque para prematuridade, baixo peso ao nascer, idade e escolaridade materna.

Conclusões: Artigos sobre o tema são escassos e dentre os fatores de risco identificados, a maioria é passível de modificação em ações relacionadas ao cuidado pré-natal e educação em saúde.

Palavras chave: Recém-nascido, fatores de risco, programas e vigilância.

\section{RESUMEN}

Objetivo: Identificar los programas brasileños de vigilancia de recién nacidos de riesgo de mortalidad infantil, destacando las estrategias de funcionamiento y los criterios para inclusión.

Método: Revisión integrativa de la literatura en la base de datos Medical Literature Analysis and Retrieval System Online, Literatura Latino-americana y del Caribe en Ciencias de la Salud y en el portal de revistas electrónicas Scientific Eletronic Library Online. Fueron seleccionados los artículos que abordaban la descripción de los criterios de inclusión en programas de vigilancia de recién nacido de riesgo y el funcionamiento de los mismos. No fueron incluidos los editoriales o cartas al editor, artículos de opinión, resúmenes presentados en anales, tesis o disertaciones.

Resultados: Diecisiete artículos describieron la experiencia de ocho programas, en cuatro estados brasileños, de las regiones Sur y Sudeste. La identificación de los recién nacidos fue a través de entrevistas en las maternidades o de la Declaración de Nacidos Vivos. Los criterios utilizados incluían riesgos biológicos $\mathrm{y}$ sociales, con evidencia para precocidad, bajo peso al nacer, edad y escolaridad materna.

Conclusiones: Los artículos sobre el tema son escasos y entre los factores de riesgo identificados, la mayoría es susceptible de modificación en acciones asociadas al cuidado pre-natal y educación en salud.

Palabras clave: Recién nacido, factores de riesgo, vigilancia em salud pública. 


\section{INTRODUÇÃO}

A Política de Atenção à Saúde da Mulher e da Criança, no Brasil, visa assegurar ações de promoção da saúde e prevenção de agravos, de modo a diminuir a mortalidade materna e infantil, assegurando assim, o desenvolvimento saudável das futuras gerações. A mortalidade infantil é um dos indicadores mais sensíveis para avaliar a situação de saúde e a condição social da população (Frias, Mullachery \& Giugliani, 2009) tendo sido objeto de ações dirigidas à sua redução, pelo menos, desde a segunda metade do século XX. No Brasil, essas políticas públicas receberam particular destaque com a pactuação dos Objetivos de Desenvolvimento do Milênio, os quais propunham a redução da mortalidade infantil em dois terços entre os anos de 1990 e 2015 (ONU, 2000). Esta meta foi alcançada pelo país em 2011, com um coeficiente de 15,3 óbitos por 1000 nascidos vivos (Datasus, 2015; Ipea, 2014).

Estudos epidemiológicos identificaram os principais fatores associados à mortalidade infantil e subsidiaram uma estratégia de atenção denominada "enfoque de risco", utilizada na programação dos serviços e no planejamento das ações em saúde (Cesar, 1995; Luhm, 2001). Em 2004, a publicação da agenda de compromissos visando à saúde integral da criança recomendou a vigilância do recém-nascido de risco, tendo como uma das linhas prioritárias a garantia do nascimento saudável. Considera risco para mortalidade os recém-nascidos filhos de mães adolescentes ( $<18$ anos), com baixa escolaridade ( $<8$ anos de estudo), residentes em área de risco, com história de morte de crianças menores de cinco anos na família, Os riscos atribuídos aos recém-nascidos incluem baixo peso ao nascer $(<2500 \mathrm{~g})$, prematuridade $(<37$ semanas de idade gestacional), asfixia grave no nascimento
(Apgar $>7$ no 5. ․ㅡㄴ minuto de vida), internação ou intercorrências na maternidade ou em unidade de assistência ao recém-nascido (Brasil, 2004; 2011). Apesar do aparente consenso quanto a relevância dos programas de vigilância de situações de risco e de vulnerabilidade dos recémnascidos, existem importantes lacunas em sua identificação e avaliação, tanto de suas estratégias operacionais, quanto de sua efetividade. Ainda, considerando a escassez de estudos sobre suas características e dinâmica organizacional, este artigo tem por objetivo identificar os programas brasileiros de vigilância ao recém-nascido de risco, destacando as estratégias de operacionalização e os critérios para inclusão.

\section{MÉTODO}

A presente pesquisa bibliográfica segue os pressupostos da revisão integrativa, que resgata estudos já publicados, os sistematiza e permite realizar análise, discussão e conclusões sobre um tema de interesse, com o propósito de aumentar a confiabilidade e profundidade das revisões. $\mathrm{O}$ processo seguiu os seguintes passos: seleção do tema e das palavras chaves; definição da base de dados para busca; estabelecimento dos critérios para seleção da amostra; identificação do panorama geral do resultado da busca; construção de um formulário para registro dos dados coletados e análise dos dados e interpretação dos resultados (Ganong, 1987). A pesquisa foi realizada em julho de 2014, na base de dados Literatura Latino-americana e do Caribe em Ciências da Saúde (LILACS), no portal de revistas eletrônicas Scientific Eletronic Library Online (SCIELO) e no Medical Literature Analysis and Retrieval System Online (MEDLINE) a partir do motor 
de busca Pubmed.

As publicações localizadas, no LILACS e no SCIELO, utilizaram os seguintes Descritores em Ciências da Saúde (DeCS), da Biblioteca Virtual em Saúde (BVS): recém-nascido, risco, programa, fatores de risco, mortalidade infantil, vigilância, grupos de risco, atenção primária à saúde, programas e avaliação de programas e projetos de saúde. Para a busca, os descritores recém-nascido, risco e programas foram associados com $\mathrm{O}$ operador boelano "OR", resultando em 24493 referências no LILACS e 19194 referências no SCIELO. Essas foram associadas, individualmente, através do operador boleno "AND" com os descritores: fatores de risco, mortalidade infantil, vigilância, grupos de risco, atenção primária, morbimortalidade infantil e projeto de saúde. Com essa estratégia foram encontradas 4199 referências no LILACS e 1848 no SCIELO. No MEDLINE foram associados os Medical Subject Headings (MESHS): Infant, Newborn e Infant Mortality resultando em 575219 referências. Após, esses resultados foram associados, individualmente, através do operador boleano "AND" com os MESHs: Government Programs; Infant Mortality; Public Health Surveillance e Program Evaluation, resultando em 60347 referências. Por último, os resultados foram associados através do operador boleano "OR" com o MESH Primary Health Care, resultando em 4511 referências.

Após as buscas, foram incluídos os artigos que abordavam na introdução, metodologia ou resultados a descrição dos critérios de inclusão nos programas de vigilância ao recém-nascido de risco e a operacionalização dos mesmos. Foram considerados como critérios de exclusão os editoriais ou cartas ao editor, artigos de opinião, resumos apresentados em anais, teses ou dissertações. Dessa forma, 381 referências foram recuperadas no SCIELO e no LILACS e 1242, na busca realizada no MEDLINE. Para a seleção dos estudos não foi delimitado o período de publicação.

A próxima etapa consistiu em realizar a leitura dos títulos e dos resumos das referências selecionadas em todas a bases, resultando assim no descarte das referências da base MEDLINE e em 48 referências proveniente do LILACS e SCIELO. Assim, procedeu-se a leitura do texto na íntegra desses 48 artigos, sendo que 33 deles foram excluídos por não responderem ao objetivo desse estudo e quatro por tratarem-se de duplicatas, restando então, 11 artigos.Com vistas a qualificar os resultados e ampliar o campo de revisão e o corpus da análise, foram incluídas também seis referências citadas nos 11 artigos selecionados e que respondiam integralmente aos critérios de inclusão, porém não apareceram nas buscas realizadas nas bases de dados. Ao final de todo o processo de seleção foram selecionados 17 trabalhos, os quais compuseram o corpus do estudo. Depois de selecionados os estudos, realizou-se nova leitura, na íntegra, de todos os trabalhos capturados e, posteriormente, a sistematização dos achados e categorização, de acordo com a autoria, o ano de publicação, município, nome do programa, ano de implantação, operacionalização, fatores de risco utilizados para inclusão no programa de vigilância e as formas de monitoramento. Após, os dados foram agrupados em duas categorias: Estratégias de operacionalização dos programas e Critérios para inclusão dos recém-nascidos nos programas. Os fatores de risco de cada programa foram analisados e comparados com os manuais operativos do Ministério da Saúde (MS). 


\section{RESULTADOS}

Dos 17 trabalhos selecionados todos eram artigos oriundos de pesquisas publicadas entre os anos de 1992 e 2013, sendo os tipos de estudos alternaram entre descritivos, transversais, de avaliação de programa ou de coorte. A maioria das publicações possuía o enfermeiro como primeiro autor (Machado, 2003; Rossetto \& Pizzo, 2007; Paiva, Anselmi \& Santos, 2002; Tonini, Tomazzoni, Maraschin \& Schneider, 2002; Manoel, Carandina \& Giarola, 2006; Luque, Lima, Carvalhaes, Tonete \& Parada, 2011; Melo \& Uchimura, 2011; Zurita \& Uchimura, 2011; Zurita, Melo, Santana, Marcon \& Uchimura, 2010; Sassá, Higarashi, Bercini, Arruda \& Marcon, 2011; Lopes, Santander \& Marcon, 2010; Barreto, Silva \& Marcon, 2011) com maior destaque para as publicações oriundas de estudos do estado do Paraná (PR) (Rossetto \& Pizzo, 2007; Paiva et al., 2002; Tonini et al., 2002; Melo \& Uchimura, 2011; Zurita \& Uchimura, 2011; Zurita et al., 2010; Sassá et al., 2011; Lopes et al., 2010; Barreto et al., 2011; Marques, Teston, Barreto, Furlan \& Marcon, 2010; Zani, Merino, Teston, Serafim, Tsukuda \& Marcon, 2011), principalmente da cidade de Maringá (PR) (Melo \& Uchimura, 2011; Zurita \& Uchimura, 2011; Zurita et al., 2010; Sassá et al., 2011; Lopes et al., 2010; Barreto et al., 2011; Marques et al., 2010; Zani et al., 2011). Os objetivos dos estudos variaram entre descrever os critérios de risco ao nascimento (Tonini et al., 2002, Melo \& Uchimura, 2011; Zurita \& Uchimura, 2007), avaliar o programa de vigilância do recémnascido de risco (Rosseto \& Pizzo, 2007; Paiva et al., 2002; Luque et al., 2011; Rumel, Filho, Alello, Koyama \& Rosenbaun, 1992; Baldin \& Nogueira, 2008; Goulart, Xavier, Goulart, Somarriba, Almeida \& Costa, 2003) e descrever a atenção a saúde da criança de risco no primeiro ano vida, no enfoque da
Atenção Básica e no contexto familiar (Machado, 2003; Manoel et al., 2006; Sassá et al., 2011; Lopes et al., 2010; Barreto et al., 2011; Marques et al., 2010; Zani et al., 2011).

No geral, os estudos que objetivaram avaliar o programa de vigilância utilizaram diferentes enfoques e métodos, porém a sua maioria apontou a importância do programa, assim como readequações, principalmente na atenção à criança nas Unidades Básicas de Saúde e investimentos dos gestores municipais. Do total das publicações foram identificados oito programas de vigilância de recém-nascido de risco, sendo três no estado de São Paulo, três no Paraná, um no Rio Grande do Sul e um em Minas Gerais. O município de Bauru, no estado de São Paulo (SP) foi pioneiro na implantação do programa de vigilância de recém-nascidos e desde 1986 instituiu o "Programa de Defesa da Vida dos Lactentes" (Rumel et al., 1992), seguido pelo município de Santos (SP), em 1991 (Baldin \& Nogueira, 2008).

Em Porto Alegre, estado do Rio Grande do Sul (RS) existe, desde 1992, o “Programa Pránenê" (Machado, 2003) e em Londrina (PR), foi implantado, em 1993, o "Programa de Vigilância de Recém-nascido de Risco" (Rosseto \& Pizzo, 2007). Em 1995, foi iniciado o programa "Viver em Cascavel" do município de Cascavel (PR) (Paiva et al., 2002; Tonini et al., 2002) e o "Projeto Vidaação" em Belo Horizonte, estado de Minas Gerais (MG) (Goulart et al., 2003). Em 1997, a Secretaria Municipal de Saúde de Botucatu (SP), implementou o Programa "Crescer Feliz" (Manoel et al., 2006; Luque et al., 2011) e, no ano 2000, em Maringá (PR) iniciou o "Programa de Vigilância ao Recém-nascido de Risco" (Melo \& Uchimura, 2011; Zurita \& Uchimura, 2007; Zurita et al., 2010; Sassá et al., 2011; Lopes et al., 2010; Barreto et al., 2013; Marque et al., 2010; Zani et al., 2011). Em 
relação à operacionalização, dos oito programas localizados, seis realizavam a identificação de risco através da Declaração de Nascidos Vivo (DNV) (Machado, 2003; Rosseto \& Pizzo, 2007; Paiva et al., 2002; Tonini et al., 2002; Manoel et al., 2006; Luque et al., 2011; Melo \& Uchimura, 2011; Zurita \& Uchimura, 2007; Zurita et al., 2010; Sassá et al., 2011; Lopes et al., 2010; Barreto et al., 2013; Marques et al., 2010; Zani et al., 2011; Goulat et al., 2003) e os demais por meio de entrevistas com as puérperas nas maternidades complementadas por atividades de educação em saúde, tais como orientações às mães sobre amamentação, vacinação, cuidados com o recém-nascido e incentivo ao acompanhamento na Unidade Básica de Saúde (UBS) (Rumel et al., 1992; Baldin \& Nogueira, 2008).

Quanto à classificação, a maioria dos programas incluía o recém-nascido que apresentasse um critério de risco isolado (maior risco) e/ou dois critérios de risco associados (menor risco) (Rosseto \& Pizzo, 2007; Paiva et al., 2002; Tonini et al., 2002; Manoel et al., 2006; Luque et al., 2011; Melo \& Uchimura, 2011; Zurita \& Uchimura, 2011; Zurita et al., 2010; Lopes et al., 2010; Barreto et al., 2013; Marques et al., 2010; Zani et al., 2011). Já em relação às características biológicas de inclusão nos programas foram identificados sete critérios, sendo que o peso de nascimento foi critério unânime, variando de $2500 \mathrm{~g}$ a $2700 \mathrm{~g}$. A idade gestacional entre 36 e 37 semanas também foi referida pela grande maioria dos programas (Rosseto \& Pizzo, 2007; Paiva et al., 2002; Tonini et al., 2002; Manoel et al., 2006; Luque et al., 2011; Melo \& Uchimura, 2011; Zurita \& Uchimura, 2011; Zurita et al., 2010; Lopes et al., 2010; Barreto et al., 2013; Marques et al., 2010; Zani et al., 2011).

Dois terços dos trabalhos consideravam a malformação congênita como critério de risco isolado (maior risco) (Manoel et al., 2006; Luque et al., 2011; Melo \& Uchimura, 2011; Zurita \& Uchimura, 2007; Zurita et al., 2010; Sassá et al., 2011; Lopes et al., 2010; Barreto et al., 2013; Marques et al., 2010; Zani et al., 2011; Rumel et al., 1992; Baldin \& Nogueira, 2008). O índice de Apgar do 5.. minuto foi valorizado por cinco programas, com pontos de corte diferentes (Machado, 2003; Rosseto \& Pizzo, 2007; Paiva et al., 2002; Tonini et al., 2002; Manoel et al., 2006; Luque et al., 2011; Melo \& Uchimura, 2011; Zurita \& Uchimura, 2007; Zurita et al., 2010; Sassá et al., 2011; Lopes et al., 2010; Barreto et al., 2013; Marques et al., 2010; Zani et al., 2011). Ser filho de mãe portadora de vírus da imunodeficiência humana (HIV) foi critério de risco para um terço dos programas (Manoel et al., 2006; Luque et al., 2011; Melo \& Uchimura, 2011; Zurita \& Uchimura, 2007; Zurita et al., 2010; Sassá et al., 2011; Lopes et al., 2010; Barreto et al., 2013; Marques et al., 2010; Zani et al., 2011). Ter permanecido internado ao nascer foi citado para três programas (Paiva et al., 2002; Tonini et al., 2002; Baldin \& Nogueira, 2008) e a gemeralidade foi critério considerado de risco em apenas um (Machado, 2003). Os critérios sociais totalizaram 14 itens em nove programas, com uma distribuição média de cinco critérios por programa, destacando-se a idade materna inferior a 18 anos, analfabetismo e possuir mais de três filhos vivos.

A identificação da idade materna como risco variou de 17 anos a menor de 20 anos (Machado, 2003; Rosseto \& Pizzo, 2007; Manoel et al., 2006; Luque et al., 2011; Melo \& Uchimura, 2011; Zurita \& Uchimura, 2007; Zurita et al., 2010; Sassá et al., 2011; Lopes et al., 2010; Barreto et al., 2013; Marques et al., 2010; Zani et al., 2011; Rumel et al., 1992; Goulart et al., 2003), e um dos programas acrescentou idade igual ou superior a 40 
anos (Paiva et al., 2002; Tonini et al., 2002). Ser analfabeta foi critério para a maioria dos programas (Machado, 2003; Manoel et al., 2006; Luque et al., 2011; Baldin \& Nogueira, 2008; Goulart et al., 2003), outros ampliaram os anos de estudo entre oito anos ou 1. ${ }^{\circ}$ grau incompleto (Paiva et al., 2002; Tonini et al., 2002; Melo \& Uchimura, 2011; Zurita \& Uchimura, 2007; Zurita et al., 2010; Sassá et al., 2011; Lopes et al., 2010; Barreto et al., 2013; Marques et al., 2010; Zani et al., 2011). Nascimento por parto domiciliar ou extrahospitalar foram considerados por quatro programas (Machado, 2002; Paiva et al., 2002; Tonini et al., 2002; Manoel et al., 2006; Luque et al., 2011; Melo \& Uchimura, 2011; Zurita \& Uchimura, 2007; Zurita et al., 2010; Sassá et al., 2011; Lopes et al., 2010; Barreto et al., 2013; Marques et al., 2010; Zani et al., 2011). Possuir três filhos ou mais foi critério citado em três programas (Paiva et al., 2002; Tonini et al., 2002; Manoel et al., 2006; Luque et al., 2011; Melo \& Uchimura, 2011; Zurita \& Uchimura, 2007; Zurita et al., 2010; Sassá et al., 2011; Lopes et al., 2010; Barreto et al., 2013; Marques et al., 2010; Zani et al., 2011), e outros dois indicavam ter dois ou mais filhos menores de quatro anos (Rumel et al., 1992; Baldin \& Nogueira, 2008). História de óbito de outros filhos foi critério em um terço dos programas (Paiva et al., 2002; Tonini et al., 2002; Manoel et al., 2006; Luque et al., 2011; Melo \& Uchimura, 2011; Zurita \& Uchimura, 2007; Zurita et al., 2010; Sassá et al., 2011; Lopes et al., 2010; Barreto et al., 2013; Marques et al., 2010; Zani et al., 2011). Já o chefe de família sem emprego, mãe sem companheiro (Baldin \& Nogueira, 2008) e baixas condições socioeconômicas representavam risco para dois programas (Manoel et al., 2006; Luque et al., 2011; Melo \& Uchimura, 2011; Zurita \& Uchimura, 2007; Zurita et al., 2010; Sassá et al., 2011; Lopes et al., 2010; Barreto et al., 2013; Marques et al.,
2010; Zani et al., 2011; Rumel et al., 1992).

Com relação às consultas de pré-natal, três programas consideraram o número de consultas, variando entre três (Rumel et al., 1992), quatro (Manoel et al., 2006; Luque et al., 2011) e seis (Machado, 2002). Apenas um programa considerava a presença de distúrbio psiquiátrico da mãe como risco para o recém-nascido (Machado, 2003). E dois avaliavam pais dependentes químicos como risco (Manoel et al., 2006; Luque et al., 2011; Melo \& Uchimura, 2011; Zurita \& Uchimura, 2007; Zurita et al., 2010; Sassá et al., 2011; Lopes et al., 2010; Barreto et al., 2013; Marques et al., 2010; Zani et al., 2011).

Após identificação de risco, o acompanhamento previa um trabalho articulado com a rede de atenção primária à saúde, na qual a criança teria atendimento prioritário. Foram identificadas duas formas de encaminhamento, uma delas, poderia ser através do agendamento da primeira consulta do recém-nascido na UBS de referência (Rosseto \& Pizzo, 2007; Paiva et al., 2002; Tonini et al., 2002; Manoel et al., 2006; Luque et al., 2011; Melo \& Uchimura, 2011; Zurita \& Uchimura, 2007; Zurita et al., 2010; Sassá et al., 2011; Lopes et al., 2010; Barreto et al., 2013; Marques et al., 2010; Zani et al., 2011; Rumel et al., 1992; Baldin \& Nogueira, 2008; Goulart et al., 2003) da família ou então, a partir do envio de uma lista à UBS com os dados dos recémnascidos de risco de sua área de abrangência (Machado, 2003). Em seis programas, a visita domiciliar para agendamento da primeira consulta ou busca ativa para faltosos fazia parte da estratégia de acompanhamento (Machado, 2003; Rosseto \& Pizzo, 2007; Paiva et al., 2002; Tonini et al., 2002; Manoel et al., 2006; Luque et al., 2011; Melo \& Uchimura, 2011; Zurita \& Uchimura, 2007; Zurita et al., 2010; Sassá et al., 2011; Lopes et al., 2010; Barreto et al., 2013; 
Marques et al., 2010; Zani et al., 2011; Baldin \& Nogueira,2008; Goulart et al., 2003), com indicação em dois programas de ser realizada nos primeiros 10 dias de vida da criança (Manoel et al., 2006; Luque et al., 2011; Baldin \& Nogueira, 2008).

O acompanhamento, na maioria dos programas, previa intervalo mensal entre as consultas até o primeiro ano de vida (Machado, 2003; Rosseto \& Pizzo, 2007; Melo \& Uchimura, 2011; Zurita \& Uchimura, 2007; Zurita et al., 2010; Sassá et al., 2011; Lopes et al., 2010; Barreto et al., 2013; Marques et al., 2010; Zani et al., 2011), sendo que apenas um dos programas preconizava intervalo quinzenal (Manoel et al., 2006;
Luque et al., 2011). A inclusão de crianças no programa que não foram captadas ao nascimento, no primeiro ano de vida, foi citada em um dos programas (Baldin \& Nogueira, 2008). A forma de inclusão ocorria por internação hospitalar no primeiro ano de vida ou pela identificação de risco nas UBS.

A Figura 1 apresenta os sete programas que utilizaram diferentes critérios de classificação de risco dos apontados na agenda de compromisso para saúde integral da criança e redução da mortalidade infantil, no manual de atenção à saúde do recémnascido e no caderno de pré-natal de baixo risco (Brasil, 2004; 2011; 2012a).

FIGURA 1: Programas de vigilância de recém-nascido de risco:

critérios de risco de inclusão distintos dos preconizados pelo Ministério da Saúde

\begin{tabular}{|c|c|}
\hline $\begin{array}{l}\text { Programa de vigilância } \\
\text { e ano de implantação }\end{array}$ & Critérios considerados de risco ao nascer \\
\hline $\begin{array}{l}\text { Programa de defesa da vida } \\
\text { dos lactentes de Bauru, SP. } \\
1986\end{array}$ & $\begin{array}{l}\text { Malformação congênita; Dois ou mais filhos menores de quatro anos; } \\
\text { Chefe de família desempregado; Mãe sem companheiro; Pré-natal ( } \leq 3 \\
\text { consultas); Renda inferior a } 1 / 4 \text { de salário mínimo. }\end{array}$ \\
\hline $\begin{array}{l}\text { Programa de recém- } \\
\text { nascido de risco de Santos, } \\
\text { SP. } 1991\end{array}$ & $\begin{array}{l}\text { Malformação congênita; Dois ou mais filhos menores de quatro anos; } \\
\text { Chefe da família sem emprego ou mãe como "chefe" da família; Mãe } \\
\text { sem companheiro; Irmão menor de dois anos. }\end{array}$ \\
\hline $\begin{array}{l}\text { Programa Prá-Nenê de } \\
\text { Porto Alegre, RS. } 1993\end{array}$ & $\begin{array}{l}\text { Malformação congênita; Gemelaridade; Parto domiciliar; Pré-natal (<6 } \\
\text { consultas). }\end{array}$ \\
\hline $\begin{array}{l}\text { Projeto Ninar (Viver em } \\
\text { Cascavel) - Cascavel - PR. } \\
1995\end{array}$ & $\begin{array}{l}\text { Idade materna }(<20 \text { ou }>40 \text { anos); Parto domiciliar; Possuir três ou } \\
\text { mais filhos vivos; Possuir um filho morto. }\end{array}$ \\
\hline $\begin{array}{l}\text { Programa Crescer Feliz de } \\
\text { Botucatu, SP. } 1997\end{array}$ & $\begin{array}{l}\text { Malformação congênita; Filho de mãe com soropositividade para vírus } \\
\text { imunodeficiência humana; Parto extra-hospitalar; Possuir dois ou mais } \\
\text { filhos mortos; Possuir três ou mais filhos vivos; Chefe da família sem } \\
\text { emprego ou mãe como "chefe" da família; Mãe sem } \\
\text { companheiro/apoio; Pré-natal (< } 4 \text { consultas). }\end{array}$ \\
\hline $\begin{array}{l}\text { Programa de Vigilância ao } \\
\text { Recém-nascido de Risco do } \\
\text { Município de Maringá, PR. } \\
2000\end{array}$ & $\begin{array}{l}\text { Malformação congênita; Filho de mãe com soropositividade para vírus } \\
\text { imunodeficiência humana; Parto extra-hospitalar; Possuir mais de três } \\
\text { filhos vivos; Um ou mais filhos mortos; Chefe da família sem emprego; } \\
\text { Mãe sem companheiro/apoio. }\end{array}$ \\
\hline
\end{tabular}

FONTE: Elaborado pelos autores 
A malformação congênita e a gemelaridade foram os critérios biológicos acrescentados pelos programas, no entanto o maior destaque está nos critérios sociais, chegando a oito fatores diferenciais dos preconizados pelos manuais do MS. Nos programas de Londrina (Rosseto \& Pizzo, 2007) e Belo Horizonte (Goulat et al., 2003) foram encontrados um número menor de critérios e todos de acordo com o preconizado.

\section{DISCUSSÃO}

\section{Estratégias de operacionalização dos programas}

A maioria dos programas de vigilância de recém-nascido com risco para mortalidade infantil encontrados na literatura (06) foram implementados no Brasil na década de 90 e nas regiões Sul e Sudeste. Na época a taxa de mortalidade infantil era de 47,1 óbitos/1000 nascidos vivos, com importantes diferenças entre as regiões do país, sendo maior nas regiões Nordeste (75,8/1000) e Norte $(45,9 / 1000)$ (Ipea, 2014). Em relação à estratégia para a identificação dos recémnascidos de risco duas abordagens apareceram como disparadoras do processo. A primeira, e mais prevalente nas publicações, ancora-se na análise dos dados presentes na Declaração de Nascidos Vivos (DNV) e a segunda, na coleta de dados primários através de entrevistas com as puérperas na maternidade. A DNV possibilita a análise apurada do perfil demográfico dos recém-nascidos e de suas mães (Brasil, 2012a), porém apresenta limitações relacionadas à falta de dados sobre aspectos sociais, tais como: renda familiar, distúrbios psiquiátricos e uso de drogas. Um aspecto relevante que deve ser avaliado é a qualidade do preenchimento das DNV. Ao avaliar dados do Sistema de
Informações dos Nascidos Vivos (SINASC) de 2000, 2005 e 2010 foi verificada a falta de preenchimento nas variáveis número de filhos vivos $(6,4 \%)$ e número de filhos mortos $(11,9 \%)$, nas demais variáveis $\mathrm{o}$ percentual de não preenchimento ficou em torno de $5 \%$ e as ignoradas ou incorretas representaram cerca de 1,2\% em 2010 (Brasil, 2012a).

Salienta-se que as entrevistas com as puérperas, na maternidade, apresentam como vantagem os aspectos sociais que podem ser coletados de forma mais precisa e a realização de ações de educação em saúde. Esta estratégia possui algumas limitações, como por exemplo, a fragilidade materna no momento da entrevista e a falha na identificação das crianças decorrente da forma de organização do trabalho com possíveis perdas em feriados e finais de semana. Entretanto, esta estratégia, pareceu ser mais adequada pela possibilidade de interação dos entrevistadores com as puérperas e suas famílias. Todos os programas destacaram que ao apresentar risco para mortalidade, as crianças deveriam ter atendimento prioritário, com a consulta na primeira semana de vida, seja na UBS ou no domicílio, tornando-se um momento propicio para prevenir o desmame precoce, orientar a família nos cuidados à criança e reavaliar os riscos e vulnerabilidades detectadas ao nascer (Brasil, 2012b; 2012c). Nesta perspectiva, a visita domiciliar é uma importante estratégia de intervenção em situações de vulnerabilidade, experiência já consolidada no Brasil, através da Estratégia em Saúde da Família e do Programa de Agentes Comunitários. A visita domiciliar tem o potencial de produzir resultados positivos na saúde e no desenvolvimento da criança e das famílias, tais como, a redução nas taxas de fertilidade, aumento do intervalo interpartal, melhoria do vínculo 
familiar, provável redução da mortalidade infantil por causas evitáveis, além de um incremento na qualidade do cuidado materno, evitando atendimentos nos serviços de emergência, principalmente entre a população vulnerável e de menor renda (Avellar \& Supplee, 2013; Nascimento, Leite, Almeida, Almeida \& Silva, 2012).

A forma de monitoramento, descrita nos estudos, apontou as visitas domiciliares, a busca ativa de faltosos e a periodicidade das consultas como estratégias importantes, porém a maioria dos programas identificou fragilidades na construção do vínculo entre a equipe de saúde e a família dos recémnascidos de risco, indicando a necessidade de uma atenção focada na condição de risco apoiando a família para um pleno desenvolvimento destas crianças (Brasil, 2011; Machado, 2003; Manoel et al., 2006; Melo \& Uchimura, 2011; Zurita \& Uchimura, 2007; Zurita et al., 2010; Sassá et al., 2011; Lopes et al., 2010; Barreto et al., 2013; Marques et al., 2010; Zani et al., 2011).

\section{Critérios para inclusão dos recém- nascidos nos programas}

Os programas com maior número de critérios de inclusão, descritos nos estudos, foram o de Botucatu e Maringá, abrangendo além dos aspectos biológicos, várias situações de risco sociais. Dentre os critérios biológicos o baixo peso ao nascimento e a prematuridade foram indicados pela maioria dos programas, ambos são associados, na literatura, aos cuidados de saúde materno e as condições socioeconômicas desfavoráveis (Hernandez, Silva, Agranonik, Quadros \& Goldani, 2011). Nas últimas décadas houve um incremento na proporção de recém-nascidos de baixo peso no Brasil. Em 1996, o baixo peso representou $7,7 \%$, atingindo $8,5 \%$, em
2013(Datasus, 2015). Este problema está diretamente relacionado à prematuridade que, em 2011, representou 11,8\% dos nascimentos (Victora, Barros, Matijasevich \& Silveira, 2013). Entre as causas relacionadas a esse fenômeno se destaca o aumento das intervenções obstétricas e do número de nascimentos múltiplos (Victora, Aquino, Leal, Monteiro, Barros \& Szwarcwald al, 2011). Em 2010, a malformação congênita foi a terceira causa de óbitos infantis no país e a segunda nas regiões Sul e Sudeste, sendo a primeira na região Sul para óbitos no período pósneonatal (Brasil, 2012d). No entanto, no nível de programas de vigilância, sua presença pode ser subestimada, pois algumas malformações são de difícil diagnóstico ao nascimento (Neto, Zhang, Nicoletti \& Barth, 2012).

Dentre os critérios sociais se destacam a baixa escolaridade e a idade materna, a presença de mais de três filhos vivos, o parto domiciliar e o número de consultas de prénatal. No Brasil, observa-se a redução na mortalidade infantil de acordo com a escolaridade materna. Em 1996, a proporção de óbitos infantis de mães analfabetas foi de $8,8 \%$, com redução para 4\% em 2013 (Datasus, 2015c). Por outro lado, há uma estabilização na proporção de óbitos entre mulheres com maior escolaridade, apesar de sua associação com parto cesáreo, que por sua vez, pode elevar o número de recémnascidos prematuros e de baixo peso (Hernandez et al., 2011; Victora et al., 2011). Em 1996, os óbitos infantis de mães com idade igual ou superior a 35 anos representaram 5,9\% do total de óbitos em menores de um ano. Esta proporção praticamente dobrou em 2013, representando 11,2\% (Datasus, 2015c). Nas últimas décadas houve um declínio na fecundidade da população brasileira, 
segundo projeção do Instituto Brasileiro de Geografia e Estatística (Ibge, 2015) esta taxa, em 2015, era de 1,72 filhos por mulher em idade fértil, sendo que em 2000 estava em 2,39 . O número de filhos pode acarretar a divisão do cuidado materno, porém o maior problema parece não estar centrado, necessariamente, no número de filhos, mas sim no reduzido intervalo interpartal. $\mathrm{O}$ parto extra-hospitalar foi considerado como risco e apresenta tendência decrescente. Em 1996, das 75.024 crianças menores de um ano que foram ao óbito no Brasil, 16,5\% nasceram no domicilio ou em via pública, entretanto, em 2013 estes percentuais reduziram-se para 5,9\%. Essa queda significativa deve-se a um maior acesso aos serviços de saúde (Ibge 2015). A realização do acompanhamento pré-natal tem um papel fundamental na redução do número de recém-nascidos de risco. Ao se considerar um número mínimo de seis consultas prénatais, observa-se uma assistência de qualidade que permite a identificação de infecções urinárias, retardo do crescimento intrauterino, prevenção de intercorrências, entre outros agravos (Nascimento et al., 2012; Hernandez et al. 2012; Geib, Fréu, Brandão \& Nunes, 2010). Além disso, propicia orientar sobre aleitamento materno, cuidados com recém-nascidos, tipos de parto e o empoderamento das mulheres e familiares.

Dentre as variáveis socioeconômicas, a baixa renda familiar e o fato residir em área de vulnerabilidade social são fatores importantes ao se considerar o risco para mortalidade infantil. Eles estão fortemente implicados nas práticas de cuidados de saúde das crianças, principalmente quando associados ao baixo peso, prematuridade e cuidados pré-natais inapropriados (Vettore, Gama, Lamarca, Schilithz \& Leal, 2010). Os critérios para classificação de risco adotados pelos programas, em sua maioria, estão em consonância com a Agenda de Compromissos para Saúde Integral da Criança e Redução da Mortalidade Infantil (Brasil, 2004) apesar da implantação dos programas de vigilância terem sido em período anterior a publicação da agenda. No entanto, alguns programas ampliaram os critérios de risco tanto biológicos como sociais.

Em 2012, o Ministério da Saúde (Brasil, 2012a) sugeriu três novos indicadores: idade materna menor de 16 anos, soropositividade materna para vírus imunodeficiência humana, história de morte, aborto ou malformação por sífilis congênita em gestações anteriores, mães com distúrbio psiquiátrico ou usuárias de drogas que impeçam o cuidado à criança e oriundas de famílias sem fonte de renda, e a condição de criança manifestadamente indesejada. A revisão periódica dos critérios de risco, a partir da análise das causas e perfis dos óbitos infantis de cada município, amplia as possibilidades de captação e acompanhamento adequado das crianças de acordo com o perfil epidemiológico de morbimortalidade. Além disso, possibilita o esclarecimento dos elementos da cadeia de eventos determinantes do óbito, de modo a identificar as desigualdades em saúde e a realização de intervenções eficazes na redução da mortalidade infantil (Hartz, Champagne, Leal \& Contandriopoulos, 1996).

Diversas alterações nas políticas de saúde, que possibilitaram maior autonomia nas ações de saúde nos municípios, concretizaram-se nas últimas décadas tais como: o Programa de Saúde da Família, o Programa de Redução da Mortalidade Infantil e a descentralização do financiamento do Sistema Único de Saúde (Viana \& Poz, 2005). 
De um modo geral, houve redução na mortalidade infantil em todos os municípios que implantaram programas de vigilância. O município de Santos apresentava, em 1995, uma taxa de mortalidade infantil de 30,3 óbitos/1000 nascidos vivos (NV), em 2013 esta taxa foi 13,9 óbitos/1000 NV. Em Belo Horizonte a taxa era de 35 óbitos/1000 NV e reduziu para 11,1/1000 NV em 2013. Neste período, em Porto Alegre a queda foi de 10, 4 pontos percentuais (pp) e em Maringá de 2,5 pp (Portal ODM, 2014; Datasus, 2015d). Porém, não existe evidencia que estabeleça uma relação causal entre a implantação dos programas e a redução na mortalidade. Atualmente observa-se um declínio no coeficiente da mortalidade infantil, principalmente dos óbitos ocorridos no período pós-neonatal. Esse componente agrega o maior contingente de fatores de risco evitáveis. Neste sentido, a priorização da atenção à saúde dos recém-nascidos que apresentam risco ao nascer ou durante o primeiro ano de vida (Brasil, 2011) possibilita o monitoramento da ocorrência de morbidades preveníveis pela atenção básica à saúde.

Portanto, o desafio contemporâneo está no enfrentamento da mortalidade no período neonatal precoce, o qual apresenta taxas elevadas em todo território nacional. A estratégia Rede Cegonha, elaborada com intuito de assegurar uma rede de cuidados às mulheres e aos recém-nascidos, poderá qualificar a atenção do pré-natal e do parto (Brasil, 2014).

\section{CONCLUSÃO}

A revisão da literatura permitiu o resgate das experiências municipais e os nove programas apontaram as estratégias operacionais, os principais critérios de risco para monitoramento e prevenção da mortalidade infantil. A publicação em artigos técnicos científicos sobre o tema é incipiente, apesar da relevância da vigilância na identificação de riscos possibilitar uma postura proativa dos serviços de saúde.

Observou-se consonância entre os programas com relação aos fatores de risco biológicos. Dentre os riscos sociais, há necessidade de trabalhar as questões de forma intersetorial, com a diminuição do analfabetismo, do acesso ao planejamento familiar e a qualificação da atenção pré-natal e do parto de modo a evitar a prematuridade e o baixo peso.

\section{REFERENCIAS}

- Avellar, A.S. \& Supplee, L.H. (2013). Effectiveness of Home Visiting in Improving Child Health and Reducing Child Maltreatment. Pediatrics, 132 (2), 90-99. Recuperado

de http://pediatrics.aappublications.org/content/132/S upplement 2/S90.

- Baldin, P.E.A. \& Nogueira, P.C.K. (2008). Fatores de risco para mortalidade infantil pós-neonatal. Rev Paul Pediatr, 26(2),156-60. Recuperado de http://www.scielo.br/pdf/rpp/v26n2/a11v26n2.pdf.

- Barreto, M.S., Silva, R.L.D.T. \& Marcon, S.S. (2013). Morbidade em crianças menores de um ano consideradas de risco: estudo prospectivo. Braz J Nurs, 12 (1), 5-18. Recuperado de http://www.objnursing.uff.br/index.php/nursing/a rticle/view/3999.

- Cesar, C.L.G. (1995). O Enfoque de Risco na Programação em Saúde. São Paulo: Painel de debate.

- Ministério da Saúde. (2004). Agenda de compromissos para a saúde integral da criança e redução da mortalidade infantil. Brasília: Ministério da Saúde.

- Ministério da Saúde. (2011). Atenção à saúde do recém-nascido: guia para os profissionais de saúde. Brasília: Ministério da Saúde.

- Ministério da Saúde. (2012a). Atenção ao pré-natal de baixo risco. Normas e Manuais Técnicos. Brasília: Ministério da Saúde.

- Ministério da Saúde. (2012b). Monitoramento de indicadores de qualidade dos Sistemas de Informações sobre Mortalidade (SIM) e Nascidos 
Vivos (SINASC), nos anos 2000, 2005 e 2010. In: Ministério da Saúde. (2012b). Departamento de Análise de Situação de Saúde. Saúde Brasil. Uma análise da situação de saúde e a vigilância da saúde da mulher. Brasília: Ministério da Saúde.

- Ministério da Saúde. (2012c). Saúde da criança: crescimento e desenvolvimento. Normas e Manuais Técnicos. Brasília: Ministério da Saúde.

- Ministério da Saúde. (2012d). Mortalidade infantil no Brasil: tendências, componentes e causas de morte no período de 2000.Análise da situação de saúde e a vigilância da saúde da mulher. Brasília: Ministério da Saúde.

- Ministério da Saúde. (2014). Humanização do parto e do nascimento. Cadernos HumanizaSUS. Brasília: Ministério da Saúde.

- Ministério da Saúde. (2015). Departamento de informática do SUS (Datasus). Brasília: Ministério da Saúde. Recuperado de http://tabnet.datasus.gov.br/cgi/idb2012/c01b.htm.

- Ministério da Saúde. (2015b). Departamento de informática do SUS (Datasus) Brasília: Ministério da Saúde. Recuperado de http://tabnet.datasus.gov.br/cgi/deftohtm.exe?sina sc/cnv/nvuf.def.

- Ministério da Saúde. (2015c). Departamento de informática do SUS (Datasus) Brasília: Ministério da Saúde. Recuperado de http://tabnet.datasus.gov.br/cgi/deftohtm.exe?sim/ cnv/inf09uf.def.

- Ministério da Saúde. (2015d). Departamento de informática do SUS (Datasus). Brasília: Ministério da Saúde. Recuperado de http://tabnet.datasus.gov.br/tabdata/cadernos/cad ernosmap.htm.

- Frias, P.G., Mullachery, P.H. \& Giugliani, E.R.J. (2009). Políticas de saúde direcionadas as crianças brasileiras: breve histórico baseado na oferta de serviços de saúde. In: Ministério da Saúde (2009). Saúde Brasil 2008: 20 anos de Sistema único de Saúde (SUS) no Brasil. Estatísticas e Informação em saúde. Brasília: Ministério da Saúde.

- Ganong, L. H. (1987). Integrative reviews of nursing research. Res Nurs Health, 10(1), 1-11.

- Geib, L.T.C., Fréu, C.M., Brandão, M. \& Nunes, M.L. (2010). Determinantes sociais e biológicos da mortalidade infantil em coorte de base populacional em Passo Fundo, Rio Grande do Sul. Ciênc Saúde Coletiva, 15(2), 363-370. Recuperado de: http://www.scielo.br/pdf/csc/v15n2/v15n2a11.pdf.

- Goulart, L.M.H.F., Xavier, C.C., Goulart, E.M.A., Somarriba, M.G., Almeida, C.G. \& Costa, A.L.D.G. (2003). Avaliação da ação de vigilância à morbimortalidade na periferia de Belo Horizonte, Minas Gerais. Rev Saúde Matern Infant, 3(3), 291-304.
Recuperado de http://www.scielo.br/pdf/rbsmi/v3n3/17924.pdf.

- Hartz, Z.M.A., Champagne, F., Leal, M.C. \& Contandriopoulos, A.P.(1996). Mortalidade infantil "evitável" em duas cidades do Nordeste do Brasil: indicador de qualidade do sistema local de saúde. Rev Saúde Pública, 30 (4), 310-8. Recuperado de http://www.scielo.br/pdf/rsp/v30n4/5081.pdf.

- Hernandez, A.R., Silva, C.H., Agranonik, M., Quadros, F.M. \& Goldani, M.Z.(2011) Análise de tendências das taxas de mortalidade infantil e seus fatores de risco na cidade de Porto Alegre, Rio Grande do Sul, Brasil, no período de 1996 a 2008. Cad Saúde Pública, 27(11), 2188-2196. Recuperado de: http://www.scielo.br/pdf/csp/v27n11/12.pdf.

- Instituto Brasileiro de Geografia e Estatística (IBGE) Brasil em sintese. Recuperado de http://brasilemsintese.ibge.gov.br/populacao/taxas -de-fecundidade-total.

- Instituto de Pesquisa Econômica Aplicada (Ipea). (2014). Objetivos de desenvolvimento do milênio: relatório nacional de acompanhamento. Brasília: Ipea: MP, SPI. Recuperado de http://www.pnud.org.br/Docs/5 RelatorioNaciona lAcompanhamentoODM.pdf.

- Lopes, M.C.L., Santander, C.A. \& Marcon, S.S. (2010). Acompanhamento dos recém-nascidos de risco de uma Unidade Básica de Saúde de MaringáPR. Rev. RENE, 11(1), 114-24.

- Luhm, K.R. (2001). O enfoque de risco na organização de serviços de saúde materno-infantil: avaliação do modelo preditivo utilizado pelo Programa "Nascer em Curitiba Vale a Vida". [Tesis de maestria]. São Paulo: Faculdade de Saúde Pública da USP.

- Luque, A.L.F., Lima, C.M.G., Carvalhaes, M.A.B.L., Tonete, V.L.P. \& Parada, C.M.G. L. (2011). Avaliação da atenção ao recém-nascido de risco, na perspectiva de uma política pública de saúde. Rev. Latino-am. Enfermagem,19(2), 2838.

- Machado, M.E. (2003). Atenção à saúde prestada ao recém-nascido de risco no primeiro ano de vida: um estudo na perspectiva do Programa Prá-Nenê. Bol Saúde, 17 (1), 21-38.

- Manoel, C.M., Carandina, L,. \& Giarola, L.C. (2006). Programa de vigilância ao recém-nascido de risco: ações desenvolvidas no Centro de Saúde Escola de Botucatu, São Paulo. Rev Paul Pediatria, 24(3), 22732.

- Marques, F.B., Teston, E.F., Barreto, M.S., Furlan, C.R. \& Marcon, S.S. (2010). A rede social em famílias com recém-nascidos de risco durante o primeiro ano de vida: um estudo descritivo exploratório. Braz J Nurs, 9(2), 122-130.

- Melo, W.A. \& Uchimura, T.T. (2011). Perfil e processo da assistência prestada ao recém-nascido 


\section{Cultura de los Cuidados}

de risco no Sul do Brasil. Rev Bras Epidemiol, 14(2), 37- 323 .

- Nascimento, R.M., Leite, A.J.M., Almeida, N.M.G.S., Almeida, P.C. \& Silva, C.F. (2012). Determinantes da mortalidade neonatal: estudo caso-controle em Fortaleza, Ceará, Brasil. Cad Saúde Pública, 28(3), 559-572.

- Neto Siedersberger, P., Zhang, L., Nicoletti, D. \& Barth, M. F. (2012). Mortalidade infantil por malformações congênitas no Brasil, 1996-2008. Rev AMRIGS, 56 (2), 129-132. Recuperado de http://www.amrigs.org.br/revista/56-

02/original5.pdf.

- Organização das nações unidas (ONU). (2000). Resolução A/RES/55/2 de 8 de Setembro de 2000. Dispõe sobre a Declaração do Milênio.

- Paiva, N.S.T., Anselmi, M.L. \& Santos, C.B. (2002). Projeto "Viver em Cascavel": Análise do fluxo de informações. Rev Latino-am Enfermagem, 10(4): 53743.

- Portal ODM. (2014). Acompanhamento brasileiro dos objetivos do milênio. Sistema de relatórios dinâmicos dos objetivos de desenvolvimento do milênio. Recuperado de

http://www.relatoriosdinamicos.com.br/portalod $\underline{\mathrm{m} / 4 \text {-reduzir-a-mortalidade-infantil. }}$

- Rossetto, E.G. \& Pizzo, L.G.P. (2007). Avaliação do programa de vigilância do recém-nascido de londrina-Paraná. Cienc Cuid Saude, 6(2), 148-156. Recuperado de http://www.periodicos.uem.br/ojs/index.php/Cien cCuidSaude/article/view/4147.

- Rumel, D., Filho, D.C.C., Alello, S., Koyama, M. \& Rosenbaun, W. (1992). Acurácia dos indicadores de risco do Programa de Defesa da Vida dos Lactentes em região do estado de São Paulo. Rev Saúde Publica, 26(1): 6-11. Recuperado de http://www.scielosp.org/pdf/rsp/v26n1/02.pdf.

- Sassá, A.H., Higarashi, I.H., Bercini, L.O., Arruda, D.C. \& Marcon, S.S. (2011). Bebê de risco: acompanhando o crescimento infantil no primeiro ano de vida. Acta Paul Enferm. 24(4), 541-9.

- Tonini, N.S., Tomazzoni, M.I., Maraschin, M.S. \& Schneider, J.F. (2002). Identificação e análise dos fatores de risco biológicos e sociais das crianças nascidas em Cascavel-Paraná. Arq Ciênc Saúde Unipar, 6(1), 25-28.

- Vettore, M.V., Gama, S.G.N., Lamarca, G.A., Schilithz, A.O.C. \& Leal, M.C. (2010). Housing conditions as a social determinant of low birthweight and preterm low birthweight. Rev Saúde Pública, 44(6). Recuperado de http://www.scielo.br/pdf/rsp/v44n6/1739.pdf.

- Viana, A.L.D. \& Poz, M.R.D. (2005). A reforma do sistema de saúde no Brasil e o Programa Saúde da
Família. Rev. Saúde Coletiva, 15(Suplemento), 225264. Recuperado de http://www.scielo.br/pdf/physis/v8n2/02.pdf.

- Victora, C., Barros, F., Matijasevich, A. \& Silveira, M. (2013). Pesquisa para estimar a prevalência de nascimentos pré-termo no Brasil e explorar possíveis causas. Recuperado de http://www.unicef.org/brazil/pt/br_prematuridad e possiveis causas.pdf.

- Victora, C.G., Aquino, E.M.L., Leal, M.C., Monteiro, C.A., Barros, F.C. \& Szwarcwald, C.L. (2011). Saúde de mães e criança no Brasil: progressos e desafios. Lancet. Recuperado de http://www.abc.org.br/IMG/pdf/doc-574.pdf.

- Zani, A.V., Merino, M.F.G.L., Teston, E.F., Serafim, D., Tsukuda, S.M. \& Marcon, S. S. (2011). Recémnascido de risco na percepção da mãe adolescente. Rev. RENE, 12(2), 279-86.

- Zurita, R.C.M., Melo, W.A., Santana, R.G., Marcon, S.S. \& Uchimura, T.T. (2010). Análise de correspondência para avaliação dos registros das informações dos recém-nascidos de risco. Rev Gaucha Enferm, 31(4), 623-32.

- Zurita, R.C.M, \& Uchimura, T.T. (2007). Programa de vigilância ao recém-nascido de risco no município de Maringá-Paraná. 2007. Saud Pesq, 4(1), 55-60 REDACADEMICA. 